NORDITA-96/20 P

hep-ph/9604305

October 9, 2018

\title{
Nuclear Dependence of Structure Functions in Coordinate Space
}

\author{
P. Hoyer and M. Vänttinen \\ NORDITA \\ Blegdamsvej 17, DK-2100 Copenhagen Ø
}

\begin{abstract}
The momentum distributions of partons in bound nucleons are known to depend significantly on the size of the nucleus. The Fourier transform of the momentum $\left(x_{\mathrm{Bj}}\right)$ distribution measures the overlap between Fock components of the nucleon wave function which differ by a displacement of one parton along the light cone. The magnitude of the overlap thus determines the average range of mobility of the parton in the nucleon. By comparing the Fourier transforms of structure functions for several nuclei we study the dependence of quark mobility on nuclear size. We find a surprisingly small nuclear dependence $(<2 \%$ for $\mathrm{He}, \mathrm{C}$ and $\mathrm{Ca}$ ) for displacements $t=z \lesssim 2.5 \mathrm{fm}$, after which a nuclear suppression due to shadowing sets in. The nuclear effects observed in momentum space for $x_{\mathrm{Bj}} \lesssim 0.4$ can be understood as a reflection of only the large distance shadowing in coordinate space.
\end{abstract}




\section{Introduction}

The difference between the quark structure functions of nuclei and those of free nucleons, first observed in 1982 by the EMC collaboration [1], has generated considerable experimental and theoretical interest [2]. The measured nuclear structure function gives direct information about how the momentum distribution of quarks in nucleons is modified by nuclear binding effects. Several models have been proposed for such modifications, many of them based on a picture of the nuclear wave function in coordinate space, from which modifications of the momentum space distribution are surmised.

The inclusive lepton scattering measurements determine the single parton distributions, but do not constrain parton-parton correlations in a modelindependent way. Hence the inclusive data can only partially constrain theoretical models for the nuclear effect.

Here we wish to study the nuclear effects in coordinate space. The relation between quark distributions in momentum and coordinate space has been known for a long time, and involves no further model dependence than is needed for the usual QCD interpretation of the experimental structure

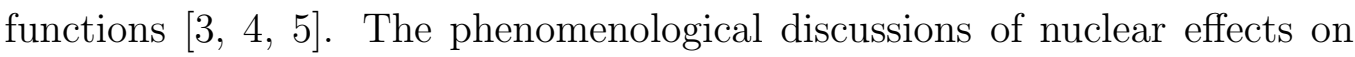
parton distributions have nevertheless concentrated almost uniquely on mo-

mentum space (see, however, Ref. [6]). While the momentum and coordinate space descriptions are in principle equivalent, insight into the physical mechanisms may benefit from viewing the quark distributions in both spaces. For example, the standard explanation of the small $x$ shadowing effect as due to the soft scattering on the nucleus of $q \bar{q}$ pairs created by the virtual photon well upstream of the target is most naturally discussed in coordinate space [0]. 
The cross section of deep inelastic lepton scattering (DIS) as a function of the photon virtuality $-Q^{2}$ and the Bjorken variable $x_{\mathrm{Bj}} \equiv x=Q^{2} / 2 m \nu$, where $m$ is the nucleon mass and $\nu$ is the energy of the photon in the target rest frame, can be parametrized as

$$
\frac{d \sigma}{d x d Q^{2}}=\frac{4 \pi \alpha^{2}}{Q^{4}} \frac{F_{2}\left(x, Q^{2}\right)}{x}\left[1-y-\frac{x y m}{2 E}+\frac{y^{2}}{2} \frac{1+4 m^{2} x^{2} / Q^{2}}{1+R\left(x, Q^{2}\right)}\right],
$$

in terms of the structure function $F_{2}\left(x, Q^{2}\right)$ and the ratio $R\left(x, Q^{2}\right)=\sigma_{L} / \sigma_{T}$ of the cross sections induced by longitudinally and transversely polarized virtual photons (in this formula, $y=\nu / E$ is the fraction of initial lepton energy carried by the photon). Measurements on a variety of nuclear targets $A$ have shown that the $A$-dependence of $R\left(x, Q^{2}\right)$ is weak [2], so that the ratio of $F_{2}$ structure functions for different targets $A_{1}, A_{2}$ is approximately given by the ratio of measured cross sections: $F_{2}^{A_{1}} / F_{2}^{A_{2}}=d \sigma^{A_{1}} / d \sigma^{A_{2}}$. The $Q^{2}$ dependence of $F_{2}^{A_{1}} / F_{2}^{A_{2}}$ is also known to be weak [2] and will not be discussed below.

According to perturbative QCD at lowest order in $\alpha_{s}$, the $F_{2}$ structure function is given by

$$
F_{2}\left(x, Q^{2}\right)=\sum_{i} e_{i}^{2} x\left[q_{i}\left(x, Q^{2}\right)+\bar{q}_{i}\left(x, Q^{2}\right)\right]
$$

where $q_{i}\left(x, Q^{2}\right)$ is a quark distribution in momentum space, i.e. the probability that a quark of flavor $i$ (having electric charge $e_{i}$ in units of $e$ ) carries a light-cone fraction $x$ of the nucleon momentum.

The deep inelastic $e N \rightarrow e^{\prime} X$ cross section is related to the forward $\gamma^{*} N \rightarrow \gamma^{*} N$ hadronic matrix element

$$
T_{\mu \nu}=\int d^{4} y \exp (i q \cdot y)\left\langle P\left|T\left[j_{\mu}(y)^{\dagger} j_{\nu}(0)\right]\right| P\right\rangle
$$


through

$$
\begin{aligned}
\operatorname{Im} T_{\mu \nu}= & 4 \pi^{2}\left[-F_{1}\left(x, Q^{2}\right)\left(g_{\mu \nu}-\frac{q_{\mu} q_{\nu}}{q^{2}}\right)\right. \\
& \left.+F_{2}\left(x, Q^{2}\right) \frac{1}{p \cdot q}\left(p_{\mu}-\frac{p \cdot q}{q^{2}} q_{\mu}\right)\left(p_{\nu}-\frac{p \cdot q}{q^{2}} q_{\nu}\right)\right],
\end{aligned}
$$

where $F_{1}=F_{2} / 2 x$ at lowest order in $\alpha_{s}$. In the frame where the virtual photon momentum is $q=\left(\nu, \overrightarrow{0}_{\perp}, \sqrt{\nu^{2}+Q^{2}}\right)$, its light-cone components $q^{ \pm} \equiv q^{0} \pm q^{3}$ are

$$
q^{+} \simeq 2 \nu, q^{-} \simeq-\frac{Q^{2}}{2 \nu}=-m x .
$$

The Fourier transform in (3) then implies a resolution in coordinate space of $\delta y^{-} \simeq 1 / 2 \nu, \delta y^{+} \simeq 1 / m x$. In the scaling limit $Q^{2}, \nu \rightarrow \infty$ with $x$ fixed, the most relevant separations $y$ between the photon currents in (3) are light-like distances of order the 'Ioffe length' $1 / 2 m x$ [8].

A visualization of $\operatorname{Im} T_{\mu \nu}$ in coordinate space is given in Fig. 1. In the target rest frame, where $P=(m, \overrightarrow{0})$, the target is moving along the $y^{0}$ axis, while the photon enters along the positive light cone. The imaginary part of $T_{\mu \nu}$ measures the overlap of two Fock states of the target, which have identical parton content except for the quark struck by the photon, whose $y^{+}$coordinate is offset by $\delta y^{+} \simeq 1 / m x$. The magnitude of the overlap is a measure of the mobility of the struck quark in the target wave function.

The precise meaning of the Fourier transform of the quark structure function is given by the operator product expansion through the relation [3, 4, 5]

$$
\begin{aligned}
& \left\langle P\left|\bar{\Psi}\left(y^{+}\right) \gamma^{-} \Psi(0)\right| P\right\rangle_{\mu^{2}} \\
& \quad=2 m \int_{0}^{A} d x\left[q\left(x, \mu^{2}\right) \exp \left(\frac{i m x y^{+}}{2}\right)-\bar{q}\left(x, \mu^{2}\right) \exp \left(-\frac{i m x y^{+}}{2}\right)\right],
\end{aligned}
$$

which here is formulated in $A^{-}=0$ gauge at a renormalization scale $\mu^{2}$ and in the rest frame of the target. The left-hand side of (6) measures ( $c f$. 
Fig. 1) the interference of target Fock states after either a quark is displaced along the light cone from 0 to $y^{+}=y^{0}+y^{3}$, or an antiquark is displaced the opposite distance. The relative minus sign is due to Fermi statistics. The kinematic upper limit of the $x$-integral on the right-hand side of (6) is the atomic number $A$ of the target, due to the scale $m=m_{N}$ used in the definition of $x$.

Experimental information on $A$-dependence is available mainly for the $F_{2}$ structure function. Subtracting from (6) the same relation with $y^{+} \rightarrow-y^{+}$ we have

$$
\begin{aligned}
& \left\langle P\left|\bar{\Psi}\left(y^{+}\right) \gamma^{-} \Psi(0)\right| P\right\rangle_{\mu^{2}}-\left(y^{+} \rightarrow-y^{+}\right) \\
& \quad=4 i m \int_{0}^{A} d x\left[q\left(x, \mu^{2}\right)+\bar{q}\left(x, \mu^{2}\right)\right] \sin \left(\frac{m x y^{+}}{2}\right) .
\end{aligned}
$$

After summing over the quark flavors weighted by $e_{i}^{2}$, the integral can be evaluated using the measured $F_{2}$ structure function for a range of targets.

\section{Numerical Analysis}

The nuclear target effects on $F_{2}$ can be expressed through the ratio

$$
R_{A}(x)=\frac{F_{2}^{A}\left(x, Q^{2}\right)}{(A / 2) F_{2}^{D}\left(x, Q^{2}\right)}
$$

of the structure function measured on a nuclear target $A$ to that on the deuteron D. Experiments show that the ratio (8) is practically independent of $Q^{2}$. There is data on $R_{A}$ for $A=\mathrm{He}, \mathrm{C}$ and Ca from both CERN [9] and SLAC [10], which together cover the ranges $0.0035<x<0.88$, $0.00015<x<0.8$ and $0.0035<x<0.8$, respectively. Measurements on heavier nuclei have been done for different choices of $A$ in different experiments, and will not be used here. The available data on $R_{C}$ is shown in Fig. 2 . 
In our evaluations of the Fourier transform (ब) we fitted the data with a smooth curve (solid line). We also used an integration algorithm based on the discrete data points to obtain an error estimate.

We used the fit of the $F_{2}^{D}$ structure function given by the NMC Collaboration [11], evaluated at $Q^{2}=5 \mathrm{GeV}^{2}$ (a typical value in experimental determinations of $R_{A / D}(x)$ ). This fit also includes SLAC and BCDMS data. The $F_{2}^{A}\left(x, Q^{2}=5 \mathrm{GeV}^{2}\right)$ structure functions are obtained as a product of the NMC fit for $F_{2}^{D}$ and our fit of the ratio (8). The Fourier transform (17) then gives the quark 'mobility' distribution in coordinate space for various nuclei,

$$
Q^{A}\left(y^{+}, Q^{2}\right) \equiv \int_{0}^{1} d x F_{2}^{D}\left(x, Q^{2}\right) R^{A}(x) \frac{\sin \left(m x y^{+} / 2\right)}{x} .
$$

Using the quark mobility distributions $Q^{A}\left(y^{+}, Q^{2}\right)$ we can then form the target ratio in coordinate space,

$$
R_{A}\left(y^{+}, Q^{2}\right)=\frac{Q^{A}\left(y^{+}, Q^{2}\right)}{(A / 2) Q^{D}\left(y^{+}, Q^{2}\right)} .
$$

Strictly speaking, the upper limit of the $x$-integral in (9) should be at $x=A$. The large $x$ region is, however, unimportant in the Fourier transform due to the small size of $F_{2}^{A}$ in this region. This is illustrated in Fig. 3, which shows the contribution to the integral in (9) from various regions of $x$. The fact that the large $x$ region is insignificant also implies that the effects of nuclear Fermi motion are suppressed in coordinate space (at moderate values of $\left.y^{+}\right)$.

From Fig. 3 one can already anticipate important cancellations of the $A$-dependence in the Fourier integral. For $y^{+}=y^{0}+y^{3}$ below $2 \mathrm{fm}$, the 'antishadowing' nuclear enhancement region beats against the EMC suppression region, while for $y^{+}$around $5 \mathrm{fm}$ anti-shadowing tends to offset the shadowing 
suppression at low $x$. At large light-cone distances the low $x$ region dominates the integral, and the shadowing effect will prevail.

Assuming a power law dependence $F_{2}^{A}(x) \simeq c_{A} x^{-\beta}$ for small $x$ gives

$$
Q^{A}\left(y^{+} \rightarrow \infty\right) \simeq c_{A}\left(\frac{m y^{+}}{2}\right)^{\beta} \int_{0}^{\infty} d u \frac{\sin u}{u^{1+\beta}} .
$$

If the power $\beta$ is independent of $A$, i.e., if $R_{A}(x)$ in (8) approaches the constant $2 c_{A} / A c_{D}$ for $x \rightarrow 0$, the coordinate space ratio $R_{A}\left(y^{+}\right)$in (10) will approach the same constant $2 c_{A} / A c_{D}$ for $y^{+} \rightarrow \infty$. This limiting value is numerically reached only for very large $y^{+}$, however (see Fig. 4 below).

In our numerical calculation, we have set $F_{2}\left(x, Q^{2}\right)=F_{2}\left(x=0.01, Q^{2}\right)$ for $x<0.01$, motivated by the $x$ range of the NMC parametrization for $F_{2}^{D}$. We have assumed the ratio $R_{A}(x)$ to approach a constant for $x \rightarrow 0$, and verified that the results presented here are insensitive to the precise value of $R_{A}(0)$ and to the behaviour of $F_{2}^{D}$ at $x<0.01$, where HERA data 12 actually show a rapid rise of $F_{2}^{D}$ with decreasing $x$.

\section{Results and Discussion}

The coordinate space target ratio $R_{A}\left(y^{+}, Q^{2}=5 \mathrm{GeV}^{2}\right)$ (10) is shown in Fig. 4 for $A=\mathrm{He}, \mathrm{C}$ and $\mathrm{Ca}$. Even for the heaviest (Ca) nucleus, the ratio is within $2 \%$ of unity for $y^{+}=y^{0}+y^{3} \lesssim 5 \mathrm{fm}$. At larger distances, the shadowing effect is clearly visible.

Fig. 5 shows error estimates for $R_{C}\left(y^{+}, Q^{2}=5 \mathrm{GeV}^{2}\right)$ derived from the actual data points and errors of the measured momentum space ratios $R_{C}(x)$ by using trapezoidal rule integration [13]. For large $y^{+}$, the finite spacing of the data points in $x$ makes any error estimate dependent on how smooth $R_{C}(x)$ is assumed to be. The result for $R_{C}\left(y^{+}, Q^{2}\right)$ shown in Fig. 4 should thus be reliable at least for $y^{+} \lesssim 7 \mathrm{fm}$. We have also verified that our results 
are insensitive to the value of $Q^{2}$ used in evaluating the structure function of the deuteron.

At low $y^{+}$, the quark mobility distribution $Q^{A}\left(y^{+}\right)$is readily seen from (9) to be proportional to $y^{+}$,

$$
Q^{A}\left(y^{+} \rightarrow 0\right) \simeq y^{+} \frac{m}{2} \int_{0}^{1} d x F_{2}^{A}(x)
$$

where the integral measures the total fraction of target momentum carried by quarks. Previous careful estimates of the $A$-dependence of this fraction [2], which took into account finite energy effects in the data, gave results compatible with no nuclear dependence,

$$
\int_{0.0035}^{0.80} d x\left[F_{2}^{\mathrm{Ca}}-F_{2}^{D}\right]=(-1.5 \pm 0.4 \pm 1.4) \cdot 10^{-3}
$$

to be normalized by $\int d x F_{2}^{D} \simeq 0.15$. As seen from Fig. 4, the deviation of $R_{\mathrm{Ca}}\left(y^{+}\right)$from unity is no larger than it is at $y^{+}=0$ in the whole region $y^{+} \lesssim 5 \mathrm{fm}$. Such a weak $A$-dependence of the structure function in coordinate space appears rather surprising, given that the EMC effect is of $\mathcal{O}(10 \ldots 15 \%)$ for $\mathrm{C}$ and $\mathrm{Ca}$ nuclei in momentum space. As discussed above, the weakness of the nuclear effect in coordinate space is due to cancellations between nuclear enhancement and suppression regions in momentum space.

Some of the models discussed in the context of nuclear effects postulated that the effective radius of a bound nucleon would be larger by up to $30 \%$ compared to that of a free nucleon ("nucleon swelling" [14). The Fourier transform (9) provides the exact relation between distributions in coordinate and momentum space. We find that a simple assumption $Q^{A}\left(y^{+}\right) \propto Q^{D}\left(\xi y^{+}\right)$ leads to an incorrect shape of the ratio $R_{A}\left(y^{+}\right)$for $y^{+} \lesssim 3 \mathrm{fm}$, as shown in Fig. 6, unless $1-\xi \lesssim 2 \%$. Such a model in any case fails to describe the shadowing region of large $y^{+}$. 
This analysis is not directly applicable to the " $Q$ 2 rescaling" models [15], where a nuclear effect arises because the effective value of $Q^{2}$ is taken to be different for bound and free nucleons due to their different radii.

Fig. 4 suggests that ignoring a possible $\lesssim 2 \%$ effect for $y^{+} \lesssim 5 \mathrm{fm}$, the main nuclear effect in coordinate space is the shadowing phenomenon at large $y^{+}$. The physical reason for shadowing in DIS is well understood at a qualitative level, and quantitative models have been successfully constructed [16]. It may thus be of some interest to see how much of the structure in momentum space can be ascribed solely to shadowing in coordinate space. This can be studied be evaluating the transform inverse to the one in (9),

$$
\widetilde{F}_{2}^{A}(x)=\frac{m}{\pi} x \int_{0}^{\infty} d y^{+} \widetilde{Q}^{A}\left(y^{+}, w\right) \sin \left(m x y^{+} / 2\right)
$$

using a function $\widetilde{Q}^{A}\left(y^{+}, w\right)$ defined as

$$
\widetilde{Q}^{A}\left(y^{+}, w\right)= \begin{cases}Q^{D}\left(y^{+}\right), & y^{+}<w, \\ Q^{A}\left(y^{+}\right), & y^{+} \geq w,\end{cases}
$$

where $w=\mathcal{O}(5 \mathrm{fm})$. Substituting the definition (9) of $Q^{D, A}\left(y^{+}\right)$and interchanging the order of integration one obtains an expression suitable for numerical evaluation,

$$
\begin{aligned}
\widetilde{F}_{2}^{A}(x, w)= & F_{2}^{A}(x)+\frac{x}{\pi} \int_{0}^{1} d x^{\prime}\left[Q^{A}\left(x^{\prime}\right)-Q^{D}\left(x^{\prime}\right)\right] \\
& \times\left\{\frac{\sin \left[m\left(x^{\prime}+x\right) w / 2\right]}{x^{\prime}+x}-\frac{\sin \left[m\left(x^{\prime}-x\right) w / 2\right]}{x^{\prime}-x}\right\} .
\end{aligned}
$$

The corresponding momentum-space target ratio

$$
\widetilde{R}_{A}(x, w)=\frac{\widetilde{F}_{2}^{A}(x, w)}{(A / 2) F_{2}^{D}(x)}
$$

for two choices of $w$ is compared with $R_{A}(x)=\widetilde{R}_{A}(x, w=0)$ in Fig. 7 . It can be seen that the nuclear effects observed in the data for $x \lesssim 0.4$ 
(which includes the anti-shadowing enhancement and the beginning of the EMC suppression) can be obtained by assuming no other nuclear effect in coordinate space than the shadowing for $y^{+} \gtrsim 5 \mathrm{fm}$. The nuclear effects at larger values of $x$ do, however, depend sensitively also on the small $(\lesssim 2 \%)$ effects suggested by the data at light-cone distances $y^{+} \lesssim 5 \mathrm{fm}$.

\section{Summary}

We have studied the nuclear effects on parton distributions in coordinate space by Fourier transforming the measured momentum space $\left(x_{\mathrm{Bj}}\right)$ distributions. Parton distributions in coordinate space can be rigorously defined using the Operator Product Expansion. Intuitively, they measure parton mobility in the target wave function, in terms of an average overlap between wave function components where one parton has been offset the given distance along the light cone.

The parton distributions at large light-cone distances $y^{+}$are sensitive only to the momentum distribution at small $x_{\mathrm{Bj}}$, and thus reflect the wellknown shadowing phenomenon. For $y^{+} \lesssim 5 \mathrm{fm}$ (i.e., for $y^{0}=y^{3} \lesssim 2.5 \mathrm{fm}$ ), on the other hand, we found the nuclear effects to be surprisingly weak $(<2 \%$ for $A=\mathrm{He}, \mathrm{C}$ and $\mathrm{Ca}$ ). Numerically, this is due to cancellations in the Fourier integral between regions of nuclear enhancement (anti-shadowing) and suppression (shadowing, EMC effect). Conversely, the observed nuclear dependence in momentum space reflects the mixing of effects from long and short distances. If the small nuclear effect at moderate light-cone distances is interpreted as an effective 'swelling' of the nucleons in the nucleus, only a $\lesssim 2 \%$ increase in the nucleon radius can be accomodated.

Through an inverse transform we verified that the nuclear effects ob-

served for $x_{\mathrm{Bj}} \lesssim 0.4$ can be obtained solely from the shadowing phenomenon 
at $y^{+} \gtrsim 5 \mathrm{fm}$ in coordinate space. The effects seen at larger $x_{\mathrm{Bj}}$ depend sensitively also on weak nuclear dependencies at $y^{+} \lesssim 5 \mathrm{fm}$.

\section{Acknowledgements}

We would like to thank V. Braun and H. Pirner for helpful discussions.

\section{References}

[1] J. J. Aubert et al., EMC Coll.: Phys. Lett. 105B (1982) 322.

[2] M. Arneodo: Phys. Rep. 240 (1994) 301.

[3] J. C. Collins, D. E. Soper: Nucl. Phys. B194 (1982) 445.

[4] I. I. Balitsky, V. M. Braun: Nucl. Phys. B311 (1989) 541.

[5] V. Braun, P. Górnicki, L. Mankiewicz: Phys. Rev. D 51 (1995) 6036.

[6] C. H. Llewellyn Smith: Nucl. Phys. A434 (1985) 35c.

[7] V. Del Duca, S. J. Brodsky, P. Hoyer: Phys. Rev. D 46 (1992) 931.

[8] B. L. Ioffe: Phys. Lett. 30B (1969) 123.

[9] P. Amaudruz et al., NMC Coll.: Nucl. Phys. B441 (1995) 3; M. Arneodo et al., NMC Coll.: Nucl. Phys. B441 (1995) 12.

[10] J. Gomez et al., SLAC-E139 Coll.: Phys. Rev. D 49 (1994) 4348.

[11] P. Amaudruz et al., NMC Coll.: Phys. Lett. B295 (1992) 159.

[12] M. Derrick et al., ZEUS Coll.: Z. Phys. C 65 (1995) 379; T. Ahmed et al., H1 Coll.: Nucl. Phys. B439 (1995) 471. 
[13] We used the TRAPER subroutine from the CERN MATHLIB library.

[14] J. V. Noble, Phys. Rev. Lett. 46 (1981) 412; A. W. Hendry, D. B. Lichtenberg and E. Predazzi, Phys. Lett. 136B (1984) 433.

[15] O. Nachtmann and H. J. Pirner, Z. Phys. C 21 (1984) 277; R. L. Jaffe, F. E. Close, R. G. Roberts and G. G. Ross, Phys. Lett. 134B (1984) 449.

[16] For a recent review, see P. Hoyer: Interactions on Nuclei, in Proc. of the Workshop on Deep Inelastic Scattering and QCD, Paris, 24-28 April 1995, eds. J. F. Laporte and Y. Sirois, p. 127. 


\section{FIGURE CAPTIONS}

Fig. 1. A space-time picture of the imaginary part of the matrix element $T_{\mu \nu}$ of (3) for forward virtual photon-nucleon scattering.

Fig. 2. Our fit of the structure function ratio $R_{C}(x)$ for carbon compared with data. Squares: NMC data [9]; diamonds: SLAC-E139 data [10]. The solid curve is the result of a least-squares cubic spline fit with interior knots at $x=0.002,0.02,0.2$ and 0.75 and an extra "data point" $R_{C}(x=0)=0.85$ added at $x=0$, with error $\Delta R_{C}(x=0)=0.01$. The dashed curves show the effect of varying $R_{C}(x=0)$ between 0.7 and 0.95. (a) Logarithmic horizontal scale, (b) linear horizontal scale.

Fig. 3. The solid curve shows the mobility distribution $Q^{D}\left(y^{+}, Q^{2}=5 \mathrm{GeV}^{2}\right)$ for deuterium. The dotted curves show the contributions to $Q^{D}\left(y^{+}, Q^{2}\right)$ from different regions in the $x$ integral of (9).

Fig. 4. The coordinate space ratio $R_{A}\left(y^{+}, Q^{2}=5 \mathrm{GeV}^{2}\right)$ for $A=\mathrm{He}, \mathrm{C}$ and Ca as obtained by using cubic-spline fits of the $R_{A}(x)$ data ( $c f$. Fig. 2 for carbon) and the NMC parametrization of $F_{2}^{D}\left(x, Q^{2}\right)$ [11]. At very large $y^{+}, R_{A}\left(y^{+}\right)$tends to the constant value $R_{A}(x=0)$, which has here been set to $0.92,0.85$ and 0.75 for He, $\mathrm{C}$ and $\mathrm{Ca}$, respectively.

Fig. 5. An estimated error band for $R_{C}\left(y^{+}, Q^{2}=5 \mathrm{GeV}^{2}\right)$. The solid line is as in Fig. 4. The dotted lines show error estimates based on the experimental errors of $R_{C}(x)$ and trapezoidal rule integration 13 . At $y^{+} \gtrsim 7 \mathrm{fm}$, the trapezoidal rule integration becomes unreliable because of the finite spacing of the data points in $x$. 
Fig. 6. Solid curve: $R_{C}\left(y^{+}, Q^{2}=5 \mathrm{GeV}^{2}\right)$ from Fig. 4. Dashed curves: $R_{C}\left(y^{+}\right)$as obtained by setting $Q_{C}\left(y^{+}\right)=Q_{D}\left(\xi y^{+}\right)$.

Fig. 7. The momentum space ratio $\widetilde{R}_{C}\left(x, w, Q^{2}=5 \mathrm{GeV}^{2}\right)$ obtained from the inverse transform (16) using the modified coordinate space distribution $\widetilde{Q}_{A}\left(y^{+}, w\right)$ (15), in which all nuclear effects are eliminated for $y^{+}<w$. 


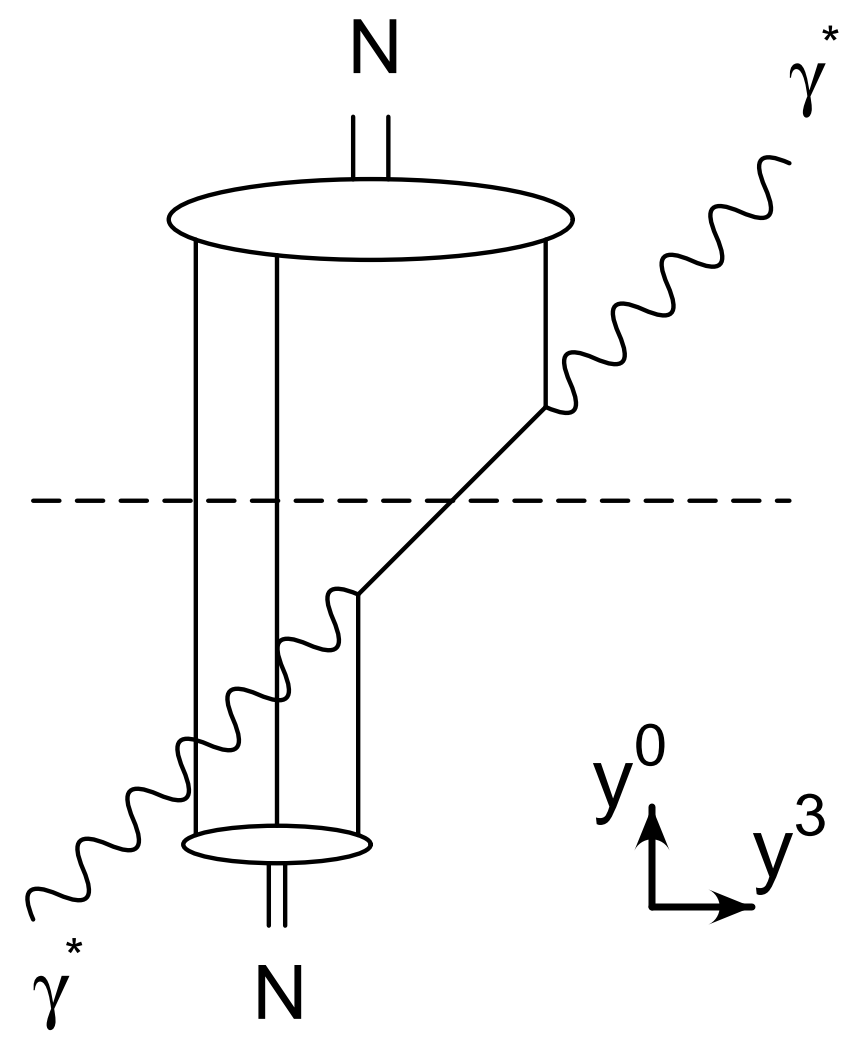




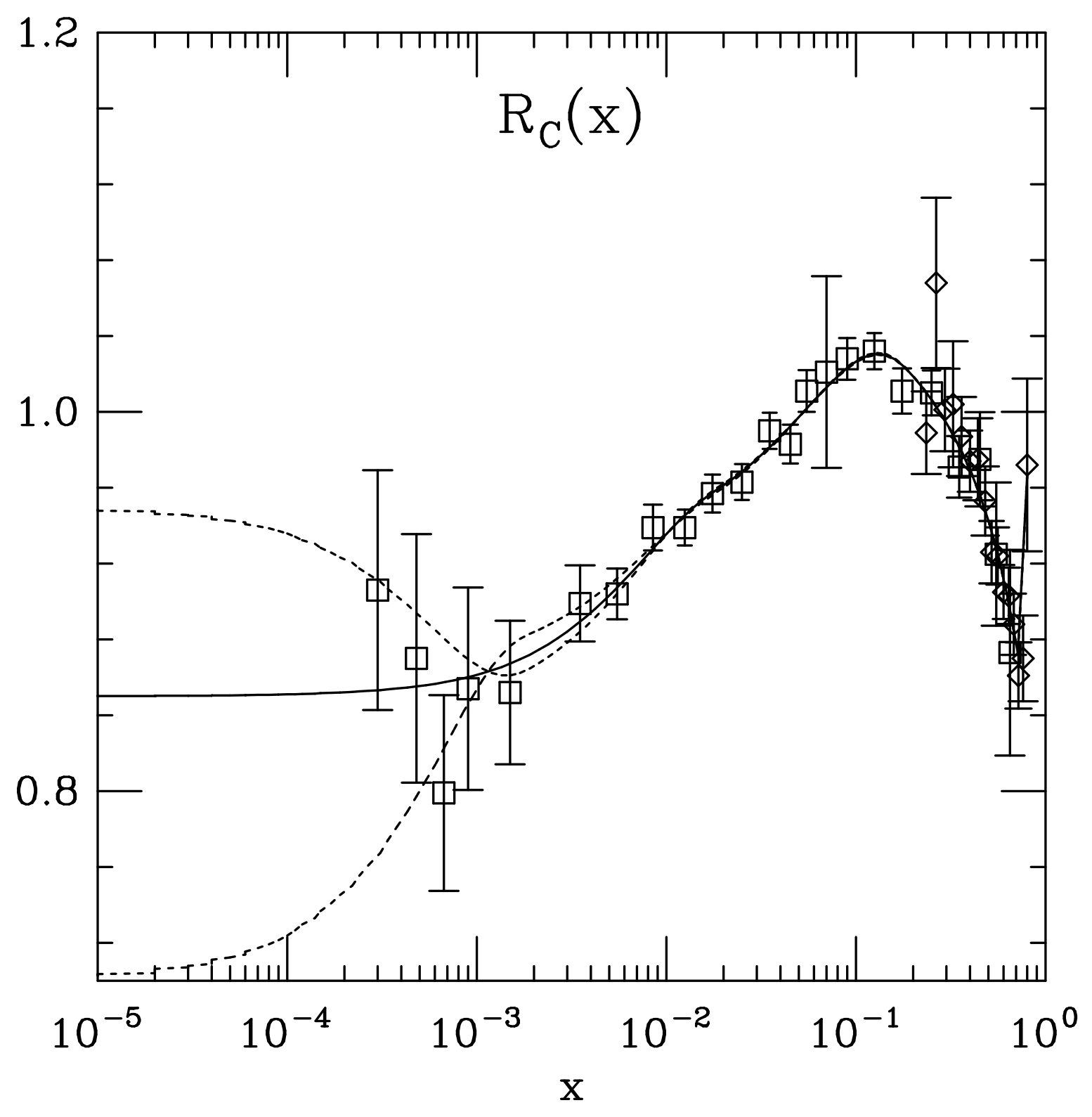




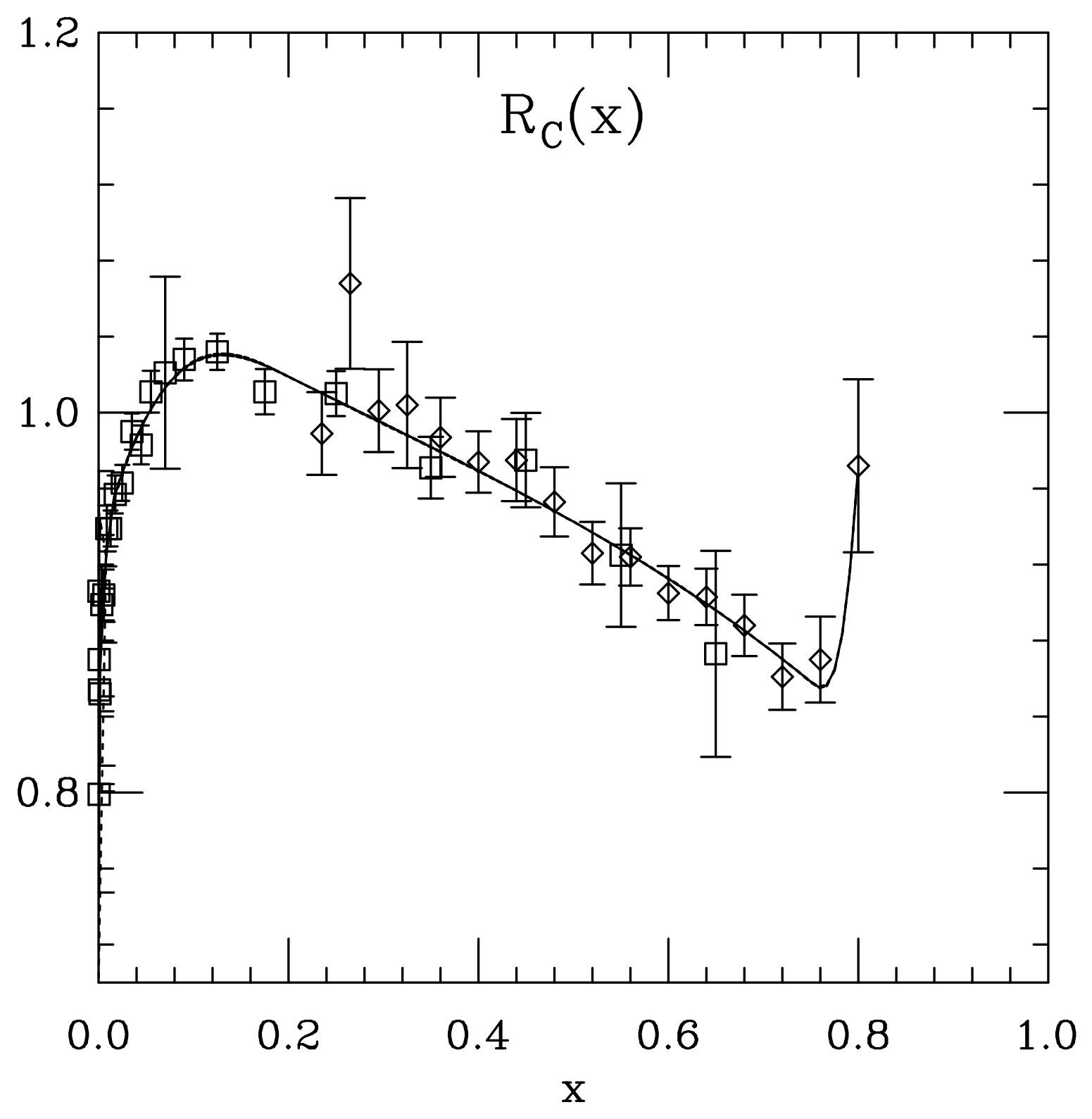




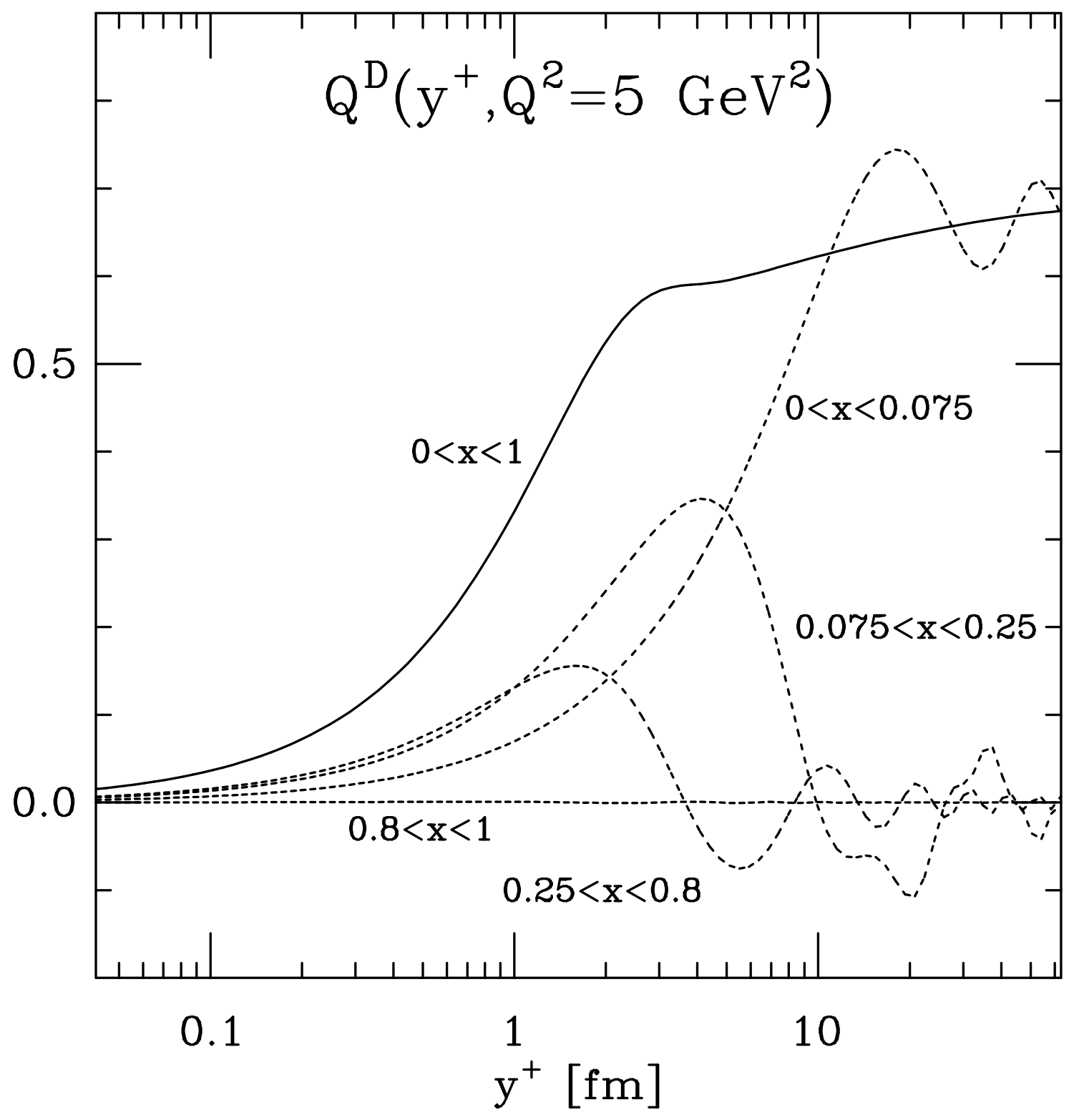




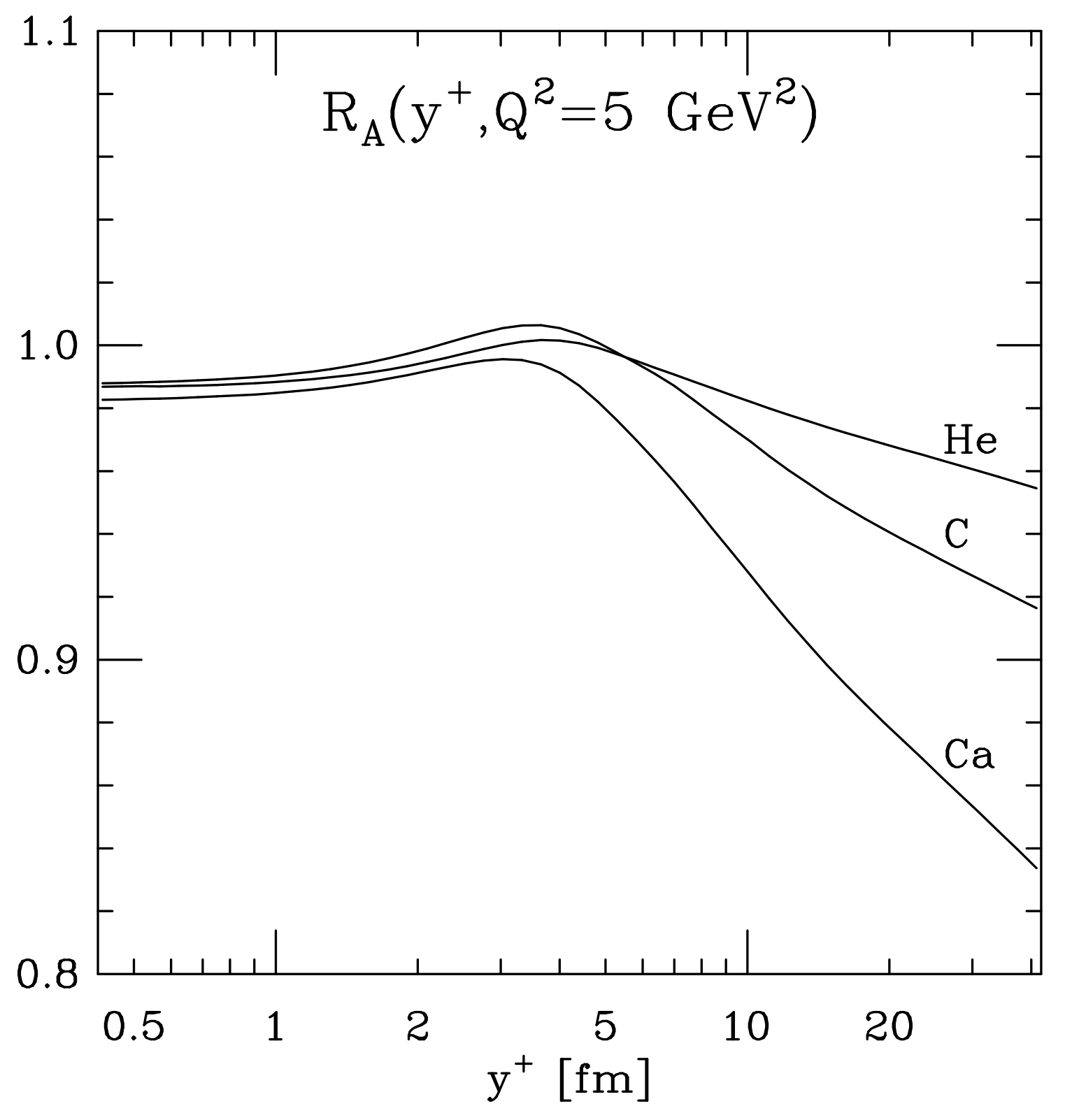




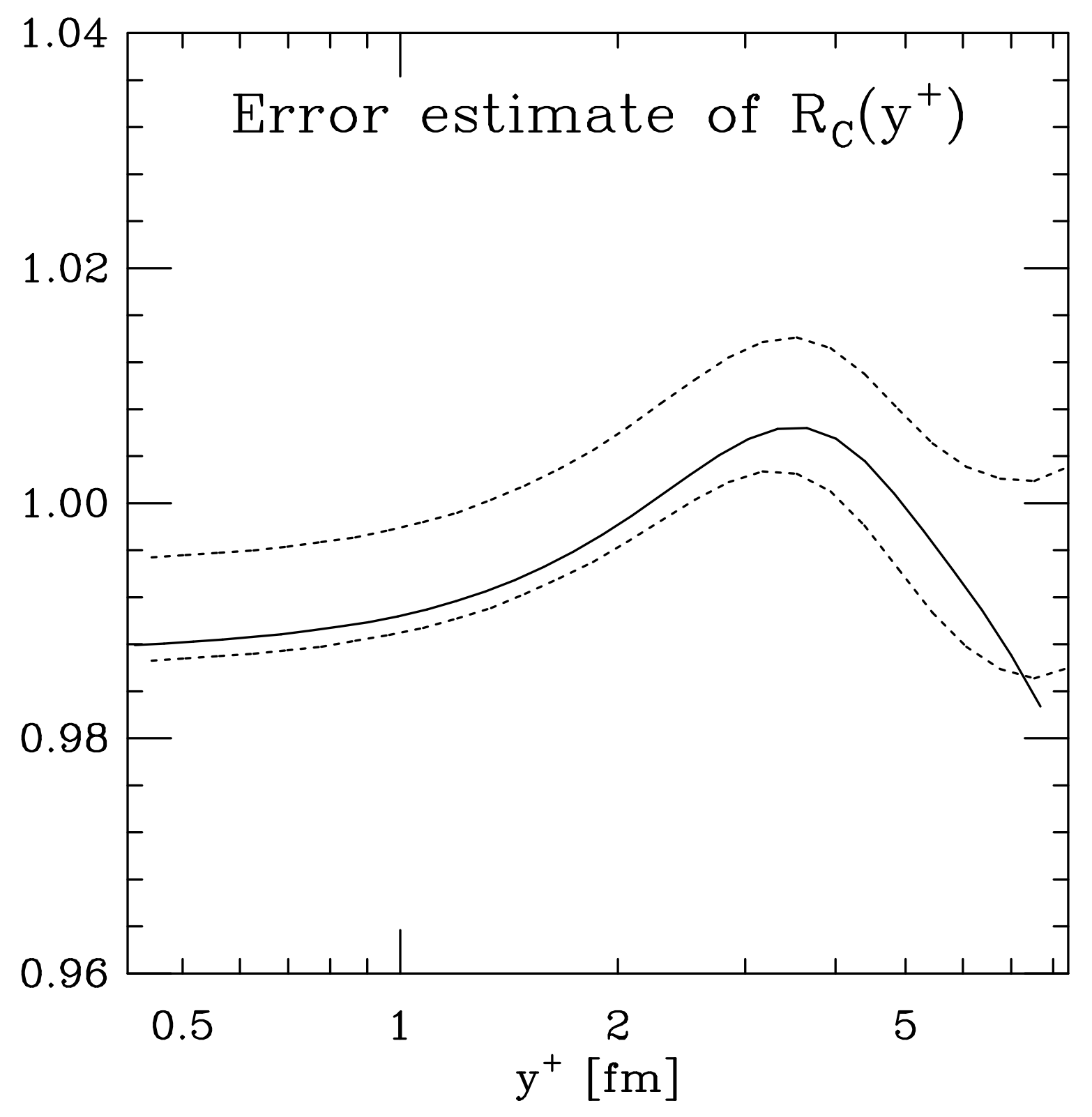




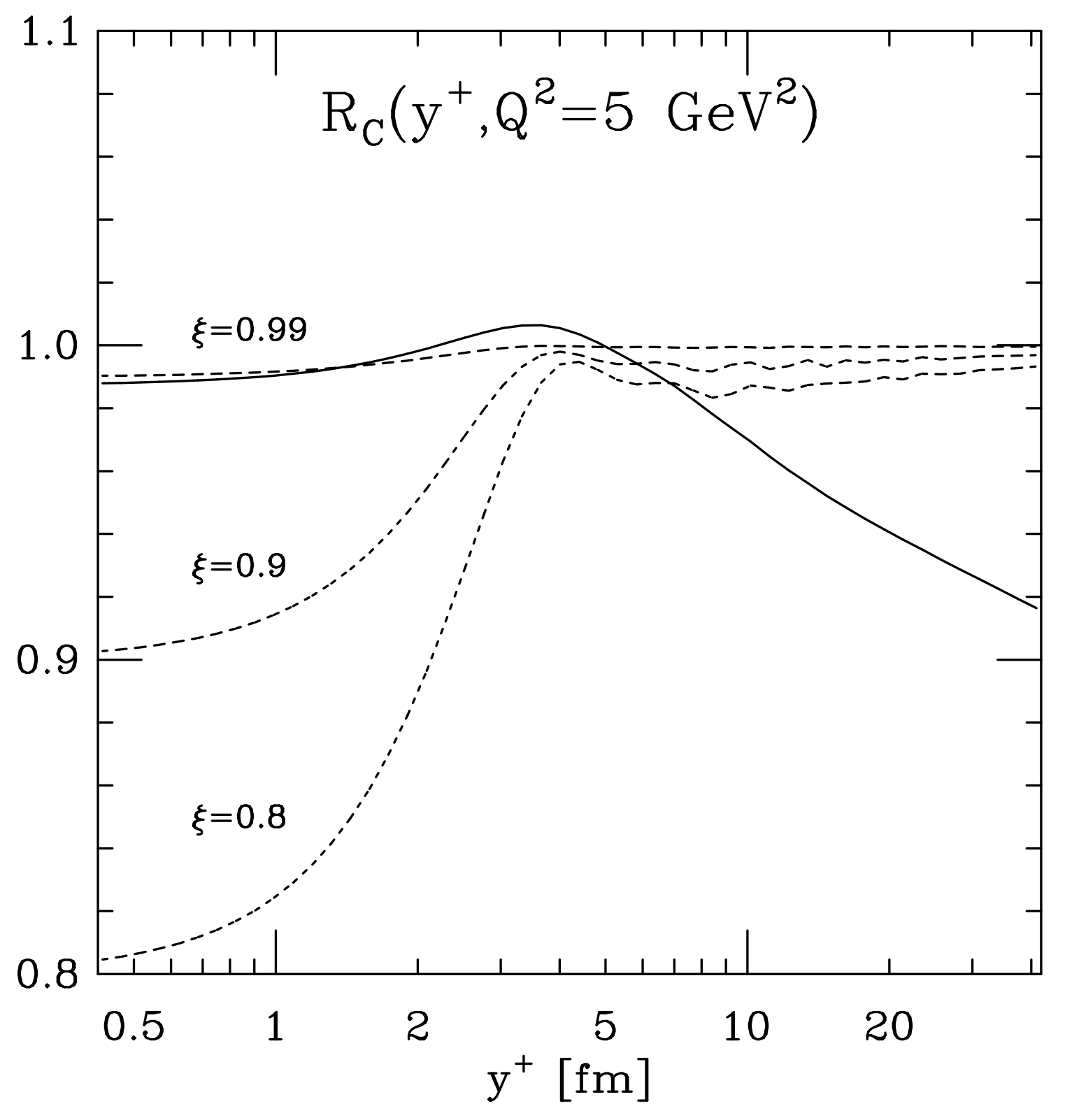




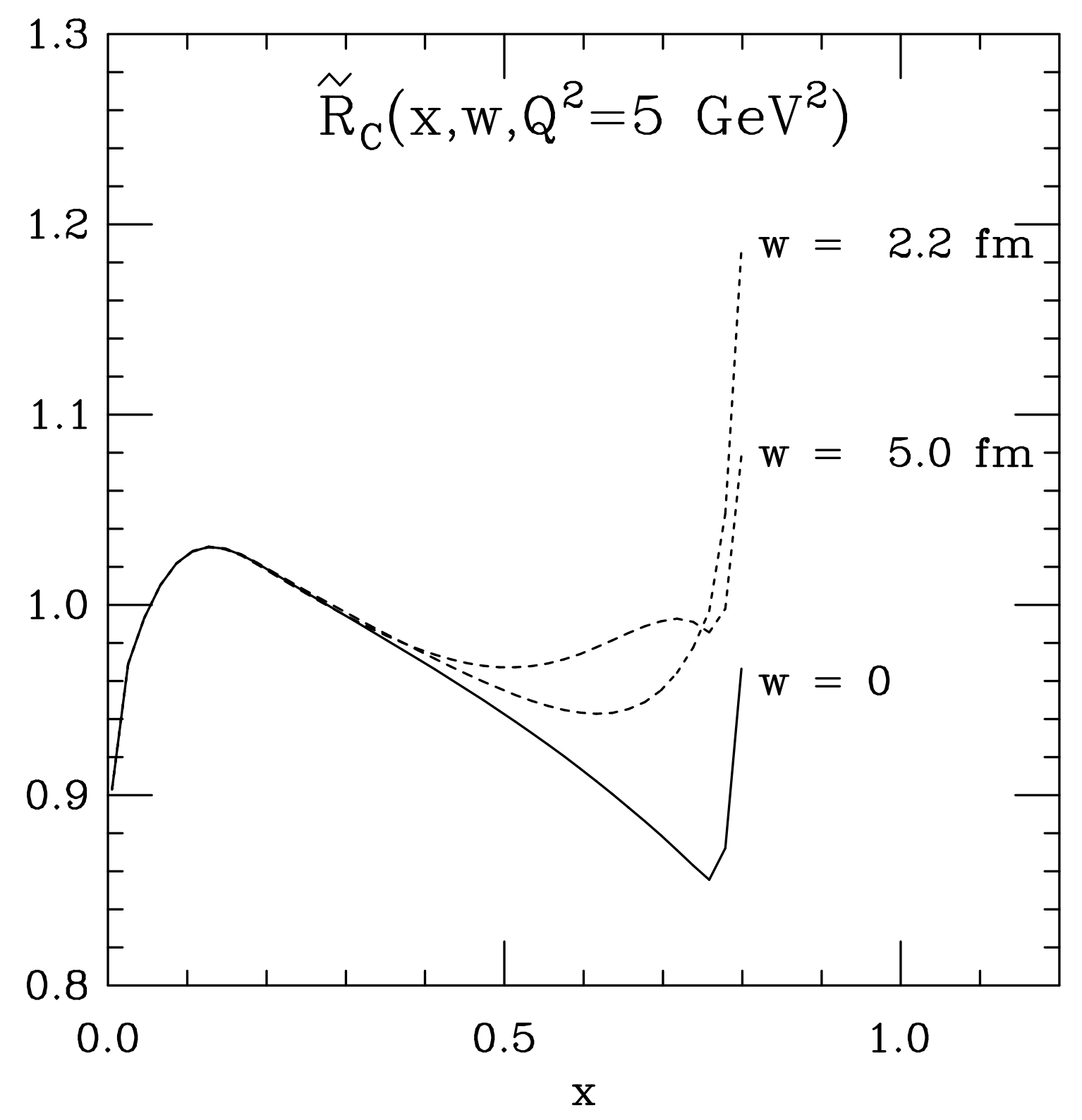

